\title{
Effects of food reward and frustrative nonreward during escape training'
}

\author{
JOSEPH J. FRANCHINA, DePaul University, Chicago, Ill. \\ 60614
}

The effects of combining two different types of reinforcers were investigated during escape training. Results showed that performance followed by both shock offset and food reward was reliably superior to that followed by either shock offset or food reward alone $(p<.005)$. When food was permanently omitted from the combination of shock offset and food (shock conditions being held constant) performance declined to a level reliably below that for shock offset alone $(p<.02)$.

Margules \& Stein (1968) stated that their finding of increased avoidance behavior under positively reinforcing brain stimulation might not occur with a more conventional positive reinforcer, such as food pellets. Presumably, under shock conditions conventional food reinforcement might lose some of its reinforcing value either through the inhibitory effects of aversive stimulation, or by the competition between the consummatory response to food (i.e., eating) and the response elicited by the aversive situation (e.g., fear). The first purpose of this experiment was to evaluate Margules and Stein's view by presenting food as a positive reinforcer in an escape from shock situation. As in the Margules and Stein study, this study used a peripheral aversive stimulus to motivate behavior but unlike the former study, the peripheral stimulus in this study was shock on all trials instead of threat of shock.

The second purpose of this experiment was to investigate the effects of frustrative nonreward on escape from shock. Amsel (1962) has assumed that the removal of previously-experienced food reward constitutes frustrative nonreward, an aversive condition with the property of punishing preceding responses. To date, the punishing effects of frustrative nonreward have been studied with approach behavior motivated by appetitive stimulation. This study investigated whether frustrative nonreward would similarly influence performance under intense aversive stimulation (i.e., shock).

\section{APPARATUS}

The apparatus has been described by Franchina (1968). Briefly, a white start box was separated from a black safe box by a guillotine door and a hurdle. The start-box floor consisted of stainless steel rods wired in an alternate-bar system for the delivery of $40-\mathrm{V}$ dc shock from a constant voltage source. The safe-box floor was Masonite and could be depressed by S's weight to act as a switch. A food cup, $3 / 4 \times 3 / 4 \times 1 / 2$ in., was affixed to the rear wall of the safe box. Illumination in each box was $7 \mathrm{ft}-\mathrm{c}$. SUBJECTS, DESIGN, AND PROCEDURES

Subjects were 48 male Holtzman albino rats. When Ss were 90-100 days old their feeding was limited to $1 \frac{1}{2}-\mathrm{h}$ access to food (ad lib) every $24 \mathrm{~h}$. On Days 5 through 8 of this feeding schedule, each $S$ explored the apparatus for $6 \mathrm{~min}$ per day and received six 45 -mg pellets with the regular daily feeding. On Day 9, Ss were randomly and evenly divided into four groups and were started in training in Phase 1 of a two-phase experiment.

Phase 1 consisted of 64 trials. Two groups (E-F and E-FX) received escape training and six food pellets in the safe-box food cup. A third group (E-NF) received escape training with no food in the food cup. The fourth group (NE-F) received no escape training but did receive six pellets in the safe-box food cup. Phase 2 consisted of 40 trials. All groups continued training under the conditions of Phase 1 except that, for the E-FX group and the NE-F group, food was permanently omitted from the food cup.

For all groups in both phases, a training trial began with the insertion of $S$ into the start box. Ten seconds later, the guillotine door was raised, activating a timer (calibrated in $.01-\mathrm{sec}$ units) and, for escape-trained groups, 40-V shock. If $S$ jumped the hurdle, the safe-box floor depressed, stopping the timer. After $3 \mathrm{sec}$ in the safe box, $S$ was removed to the home cage for an intertrial interval of $6-8 \mathrm{~min}$. If $S$ failed to jump the hurdle within $40 \mathrm{sec}, \mathrm{S}$ was removed from the start box, placed in the safe box for $3 \mathrm{sec}$, and then removed to the home cage. A latency of $40 \mathrm{sec}$ was recorded on these occasions. If any $S$ in the food groups failed to eat all the pellets in the safe box, the pellets were returned with $S$ to the home cage. Only five $S s$ (three in E-F and two in NE-F) failed to eat; this occurred only during the first 10 trials of Phase 1. Each $S$ received four trials per day in both phases. The measure of performance was reciprocal of latency of hurdle jumping.

\section{RESULTS}

Figure 1 presents mean reciprocals of latency of hurdle jumping for all groups in Phase 1. An analysis of variance over all these data yielded a reliable interaction of Treatments by Trial Blocks $(F=2.76, d f=45 / 660, p<.001)$. Simple effects analyses and Scheffé comparisons showed that, over Trial Blocks 1-4, the escape-trained groups (E-F, E-FX, and E-NF) performed similarly $(\mathrm{F}<1)$ and each performed reliably better than did the NE-F group (ps $<.001$ ). Over Trial Blocks 13-16, however, the E-F and E-FX groups performed reliably better than did the E-NF group (or the NE-F group), ps $<.005$; comparisons between E-F and E-FX and between E-NF and NE-F yielded no reliable differences ( $F s<1)$.

Figure 2 shows hurdle-jumping performance for all groups in Phase 2. The data of the NE-F group was presented just to show the effects of nonreward on appetitively-motivated behavior in the hurdle-jumping situation. These data were not employed in any statistical comparisons. The effects of frustrative nonreward and the effects of continued food presentation during escape training were evaluated with an analysis of variance over all the Phase-2 data for E-F, E-FX, and E-NF groups. A reliable Treatments by Trial Blocks

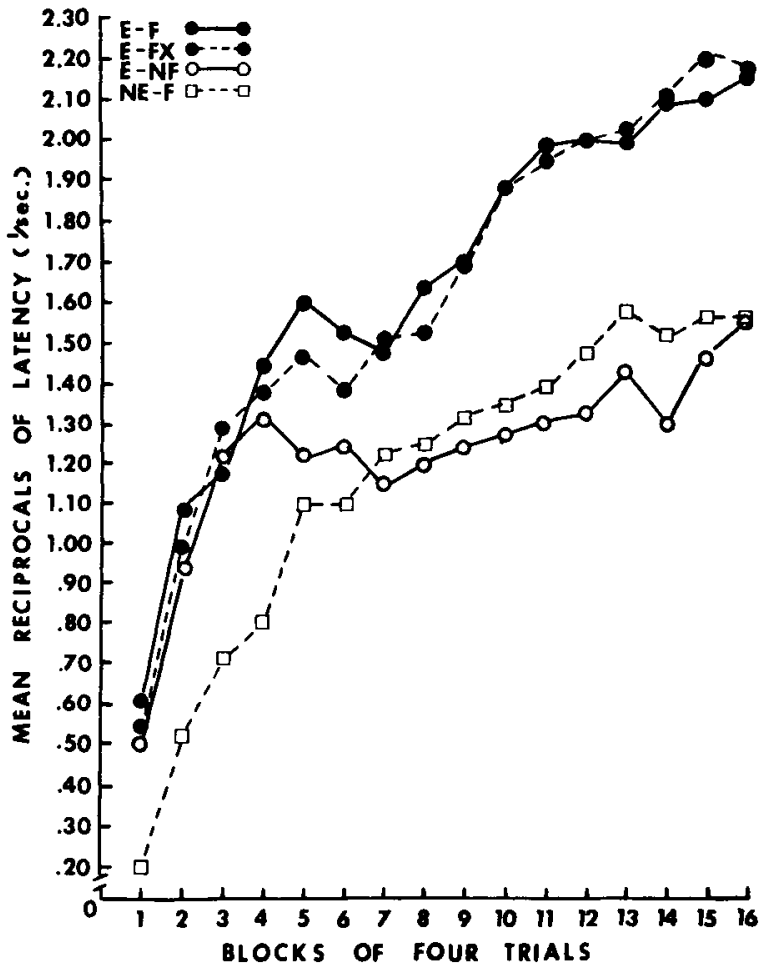

Fig. 1. Mean reciprocals of latency of hurdle jumping over blocks of four trials each in Phase 1 for groups trained with food reward alone (NE-F), with shock offset alone (E-NF) or with the combination of shock offset and food reward (E-F and E-FX). 


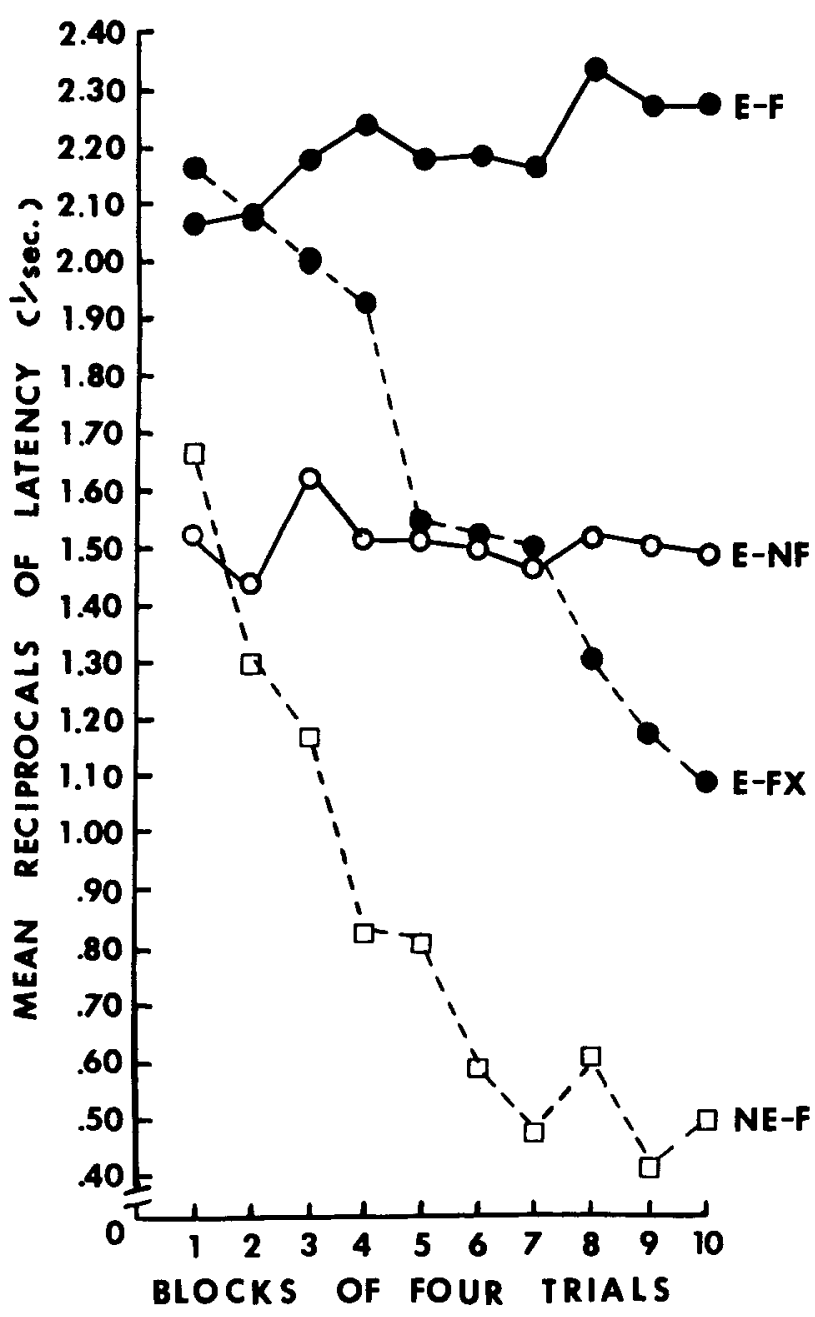

Fig. 2. Mean reciprocals of latency of hurdle jumping over blocks of four trials each in Phase 2 for E-F, E-FX, E-NF, and NE-F groups.

interaction was obtained $(F=6.70, \mathrm{df}=18 / 297, \mathrm{p}<.001)$. Simple effects analyses showed that this interaction was due to the reliable decrease in performance for the E-FX group ( $p<.001)$ over Trial Blocks 1-10, while neither the E-F nor the E-NF group showed any reliable performance change over the same span (Trial Blocks $F<1$ in each case). Scheffé comparisons between groups further showed that, on Trial Blocks 1 and 2 of Phase 2, the E-F and E-FX groups performed similarly $(F<1)$, and each was reliably superior to the E-NF group $(\mathrm{p}<.01)$. On Trial Block 10 , however, the performance of the E-FX group was reliably inferior to that of the $E-F$ and the $E-N F$ group ( $p<.001<.02$, respectively). The performance of E-F continued to be reliably superior to that of E-NF $(p<.001)$.

\section{DISCUSSION}

The results of E-F and E-NF groups for both phases showed that, in escape training, performance followed by shock offset and the presentation of a conventional positive reinforcer, food, was reliably facilitated over that followed by shock offset alone. These data were consistent with Margules and Stein's findings for positive brain stimulation during avoidance training.
It would be attractive to explain the present data by assuming that the combination of shock offset and food reward provided a greater amount of positive reinforcement for hurdle-jumping and, thereby, facilitated performance over that for shock offset alone. This explanation is consistent with the views of Margules and Stein, and Woodworth \& Schlosberg (1954). These investigators have argued that the reinforcing properties of shock offset (negative reinforcement according to Margules and Stein) are controlled by the same positive reinforcement system as that responsible for the effects of positive reinforcers like food or positive brain stimulation. Thus, instead of inhibiting negatively-reinforced behavior, positive stimulation would enhance this behavior. The results of this study were consistent with this argument.

However, the present results may have an alternate explanation. It was possible that the hurdle-jumping response of the E-F group was the result of a combination of two different response tendencies having the same behavioral index. An escape response tendency may have been reinforced by shock offset, while a separate approach tendency may have been reinforced by food reward. Since each of these tendencies provided for the performance of a hurdle-jumping response it was possible that these tendencies combined to facilitate hurdle-jumping for the E-F group over that of the E-NF group, the latter having learned only the escape response tendency. Thus, the superiority of E-F over E-NF may have been due not to increased reinforcement from two different reinforcers but to the combining of two different response tendencies.

The results of Phase 2 showed that the removal of food reward (i.e., frustrative nonreward) reliably decreased the performance of the E-FX group to a level reliably below that of the E-NF group. These data suggested that, for the E-FX group, the aversive properties of frustrative nonreward were sufficiently intense to deter hurdle-jumping performance motivated by $40-\mathrm{V}$ shock.

However, if hurdle-jumping performance for E-FX reflected both approach and escape tendencies, then one might argue that the decreasing performance of E-FX in Phase 2 was attributable to the punishing effects of frustrative nonreward on the approach tendency alone. This argument does not seem tenable since the performance of the E-FX group decreased from a level reliably above to a level reliably below that of the E-NF group. If frustrative nonreward was influencing only the approach tendency then the performance of E-FX should have declined only to the level of E-NF which had no approach training. The decline in E-FX's performance to a level reliably below that of E-NF suggests the influence of frustrative nonreward on factors other than the approach tendency alone. Perhaps these other factors were escape-from-shock tendencies.

AMSEL, A. Frustrative nonreward in partial reinforcement and discrimination learning: Some recent history and theoretical extension. Psychological Review, 1962, 69, 306-328.

FRANCHINA, J. J. Transfer of escape training. Journal of Comparative \& Physiological Psychology, 1968, 65, 175-178.

MARGULES, D. L., \& STEIN, L. Facilitation of Sidman avoidance behavior by positive brain stimulation. Journal of Comparative \& Phy siological Psychology, 1968, 66, 182-184.

WOODWORTH, R. S., \& SCHLOSBERG, H. Experimental psychology (Rev. ed.). New York: Holt, Rinehart \& Winston, 1954. NOTE

1. These data were collected at Southern Methodist University and were reported at the Southwestern Psychological Association meeting in New Orleans, 1968. 